\title{
MedienPädagogik
}

www. medienpaed.com

Zeitschrift für

Theorie und Praxis

der Medienbildung

ISSN 1424-3636

\section{Sollte uns Facebook Angst machen? \\ Medienpädagogische Arbeit und ihre Herausforderungen am Beispiel «soziale Netzwerke»}

Judith Bündgens-Kosten und Marianne Wefelnberg

\begin{abstract}
Medienpädagogische Arbeit findet in einem Spannungsfeld zwischen den häufig bewahrpädagogisch orientierten Erwartungen einzelner Akteure/-innen und den Ansprüchen, die eine mediale Umwelt an Kinder und Jugendliche stellt, statt. Gefühle, insbesondere Angst, spielen dabei, teils als bewusst eingesetztes pädagogisches Mittel, teils als unbeabsichtigter Nebeneffekt, eine grosse Rolle. Dieser Artikel beleuchtet am Beispiel "Gefahren von sozialen Netzwerken» die Rolle von Angst in der medienpädogischen Praxis, und versucht, damit verbundene Chancen und Herausforderungen zu benennen sowie mögliche Handlungsoptionen aufzuzeigen.
\end{abstract}

\section{Affektive Dimensionen medienpädagogischen Handelns}

Soziale Netzwerke spielen eine zunehmende Rolle im Medienhandeln von Kindern und Jugendlichen. Diese Entwicklung wird durch eine intensive öffentliche Diskussion über mögliche Gefahren, die von Medien ausgehen können, begleitet. Forderungen nach Verboten und Reglementierungen, aber auch solche nach einer stärkere Förderung der Medienkompetenz, finden sich gleichermassen.

Oft lässt sich beobachten, dass Diskurse über Medien und mit ihnen verbundenen Risiken und Chancen stark von Emotionen geprägt sind. Auch die Beziehung des/ der jeweiligen Einzelnen zu Medien ist nicht in emotionaler Hinsicht neutral. Entsprechend gehen die Autorinnen von der Annahme aus, dass auch die medienpädagogische Arbeit stets eine affektive Dimension besitzt, und dass diese affektive Dimension eine Rolle für die praktische medienpädagogische Arbeit - etwa an Schulen - spielt.

In diesem Aufsatz beschäftigen wir uns mit der Rolle von Angst im Zusammenhang mit der Nutzung Sozialer Netzwerke durch Kinder und Jugendliche. Andere Gefühle - etwa Scham und Ekel - sowie mit Angst verbundene Aspekte - etwa Gefahrenbewusstsein - werden ebenfalls thematisiert. Während wir uns im ersten Teil diesen Fragen auf theoretischer Ebene widmen und auf die Ergebnisse von Mediennutzungsstudien rekurrieren, werden wir uns im zweiten Teil auf die praktischen Folgen konzentrieren, genauer auf Konflikte, Missverständnisse und Schwierigkeiten, die im weitesten Sinne mit Angst oder Gefahrenbewusstsein 
zusammenhängen. Anhand von vier Problemkomplexen - Rolle von Angst und Scham, Gefahrenbewusstsein und Handlungsempfehlungen, Verzerrte Gefahrenwahrnehmung, sowie Fokus auf Extremfälle - werden wir die Probleme von Angst im Kontext medienpädagogischer Arbeit verdeutlichen, und mit Empfehlungen für die Praxis abschliessen. Der Fokus wird dabei auf dem deutschen Kontext liegen, Daten in Bezug auf andere europäische Länder werden nur am Rande mit einfliessen.

\section{Soziale Netzwerke: Gefahren und Ängste}

Technologiebezogene Ängste und Sorgen können viele Formen annehmen. Die Sorge, dass die Technologie den Menschen überflüssig machen könnte, ist genauso verbreitet wie das (manchmal leider wahre) Klischee der älteren Dame, die mit dem Fahrkartenautomaten überfordert ist - ein Bild, das für einen rapiden technologischen Wandel, mit dem die Menschen nicht mehr mitkommen, steht. Marwick (2008) spricht in diesem Zusammenhang von «Technopanics».

Technikängste in diesem allgemeinen Sinn sollen hier aber nicht im Fokus der Diskussion stehen, vielmehr werden wir uns auf einen Untertypen, auf Angst bzw. Sorge im Kontext der Sozialen Netzwerke, konzentrieren. Dabei werden wir auf die Ängste von Kindern und Jugendlichen selbst, aber auch auf die von Erwachsenen in Bezug auf das Medienhandeln von Kindern und Jugendlichen, eingehen. Angst verstehen wir hier als ein Gefühl der Bedrohung, die zwar mit einem Medium oder einem Bedrohungsszenarium konkret verbunden ist (z. B. Angst vor Cybermobbing als Phänomen), die aber nicht auf eine konkrete Gefährdungssituation bezogen ist (z. B. Furcht in einer augenblicklich ablaufenden Cybermobbing-Situation) (vgl. auch Bündgens-Kosten und Hölterhof 2013, S. 59).

Drotner (1992) bezeichnet solche Ängste gegenüber Mediengebrauch, angelehnt an Cohens (1972) Begriff der Moral panic, als «Media panics»:

From the advent of mass-circulation fiction and magazines to film and television, comics and cartoons, the introduction of a new mass medium causes strong public reactions whose repetitiveness is as predictable as the fervour with which they are brought forward. Adult experts - teachers and social workers, cultural critics and politicians - define the new mass medium as a social, psychological, or moral threat to the young (or mixtures of the three), and appoint themselves as public trouble shooters. Legal and educational measures are then imposed, and the interest lessens - until the advent of a new mass medium reopens public discussion. That spiralling motion characterises a media panic. (Drotner 1992, S. 43) 
Keuneke spricht statt von Medienpanik von Medienangst: «Zusammengefasst soll Medienangst als ein überhistorisch und -kulturell auftretendes Gefühl der Bedrohung definiert werden, das von einem Medium ausgelöst, von mehreren Mitgliedern einer Gesellschaft geteilt und durch skeptische, besorgte oder ablehnende Äusserungen zum Ausdruck gebracht wird. Die Gesamtheit dieser Äusserungen soll als Medienangstdebatte bezeichnet werden; das Objekt einer Medienangstdebatte als Angstmedium» (Keuneke 2011, S. 278f).

Die Besonderheit bei der Medienangstdebatte rund um Soziale Netzwerke ist, dass hier Mediengefahren in erster Linie als Viktimisierungspotenziale gesehen werden. Die Angst vor dem Gebrauch sozialer Netzwerke durch Kinder und Jugendliche ist oft die Angst davor, dass die physische, psychische, informationelle und finanzielle Sicherheit der soziale Netzwerke nutzenden Kinder und Jugendlichen durch Medien oder durch Menschen, die diese Medien zu eigenen Zwecken einsetzen, gefährdet wird'1

In Bezug auf Soziale Netzwerke nimmt diese grundlegende Sorge viele Formen an: Firmen, die Facebookprofile von Bewerbern/-innen ausspionieren. Pädophile Sexualstraftäter/innen auf der Suche nach Opfern (cybergrooming). Extreme politische Gruppierungen oder religiöse Fundamentalisten/-innen auf Mitgliederfang. Rassistische Inhalte und andere Formen von Hate Speech. FacebookParties. Facebook als «Datenkrake». Dumme Sprüche und unerwünschte sexuelle Anmache durch Gleichaltrige. Veröffentlichung peinlicher Partyfotos. Cybermobbing. Facebook-〈Sucht〉 und Vernachlässigung von Schule und 〈realen〉 sozialen Beziehungen. Positiv konnotierte Darstellungen von Drogenkonsum, Essstörungen, selbstverletzendem Verhalten oder Selbstmord. Zugriff fremder Personen auf private Daten und Bilder. Finanzielle Abzocke. Viren. Stalker/innen. Hacker/innen. Fakes. Einige dieser Gefährdungsszenarien - etwa Facebookparties - sind erst seit kurzem ein Thema im öffentlichen Diskurs, andere dagegen sind schon im Zusammenhang mit früheren sozialen Netzwerken wie Myspace oder SchülerVZ, oder sogar mit IRC (Internet Relay Chat), Chats oder Foren diskutiert worden.

\section{Gefahrenpotenziale aus Schüler/innensicht}

Wie schätzen nun die Schüler/innen selbst die Gefahren Sozialer Netzwerke ein? 48\% der Kinder/Jugendlichen in der deutschen Teilstichprobe der EU Kids Online Studie (Altersgruppe 8-16) gaben an, dass es im Netz allgemein Dinge gäbe, die

1 Diese Beobachtung trifft nicht auf alle Formen von Medienhandeln Jugendlicher zu, Ausnahmen sind etwa die Themen Urheberrecht/Tauschbörsen oder Hacking, sowie der gesamte Komplex "Gewaltdarstellung» (etwa in Film und Computerspiel). Hier bestehen durchaus auch Sorgen, dass Schüler/innen durch z. B. Teilnahme an Tauschbörsen selber mit dem Gesetz in Konflikt kommen. Populäre Diskurse über Gewaltdarstellungen, etwa der oft postulierten Zusammenhang zwischen Computerspielen und Gewalttaten, legen ebenfalls einen Schwerpunkt auf die Täter/innengenese: Eine Person, die diese Videospiele nutzt, so die populäre Annahme, wird zum/zur Gewalttäter/in. Nur indirekt (über die eigentlich medial beeinflusste Person vermittelt) wird hier also von einer Viktimisierung ausgegangen. 
Gleichaltrige belasten («bother) im Sinne von «made you feel uncomfortable, upset, or feel that you shouldn't have seen it.») könnten, selbst wenn nur $8 \%$ angaben, solche belastenden Dinge selber erlebt zu haben (Livingstone et al. 2011, S. 46, S. 48). Welche Risiken sehen die Schüler/innen denn konkret? Im Rahmen der JIM-Studie wurden Schüler/innen in Deutschland (Altersgruppe 12-19) zu den ihrer Meinung nach grössten Gefahren im Internet befragt. Viele der hoch bewerteten Gefahrenpotenziale spielen dabei auch bei Sozialen Netzwerken eine besondere Rolle, etwa die «Sorge vor Abzocke und Betrug» (44\%), «Angst vor Datenklau bzw. dem Missbrauch von Daten» (28\%), "Cybermobbing» (25\%), die Sorge davor, ausspioniert zu werden oder dass Zugangsdaten gehackt werden (12\%), Fotoklau/ Fotomanipulation (7\%), pädophile Personen (5\%), gefährliche Leute/Fakes (5\%), falsche Beiträge im Netz (5\%), Beleidigungen (5\%), Verletzung der Privatsphäre (5\%) (Medienpädagogischer Forschungsverbund Südwest 2010, S. 46).

Auch in der praktischen medienpädagogischen Arbeit zeigen sich Anzeichen für diese Befürchtungen. In einer Befragung von innerhalb eines Peer-Education-Projekts ausgebildeten Mulitplikatoren/-innen zeigte sich Besorgnis der Jugendlichen vor allem bezüglich der Punkte «Abzocke» im Internet, Datenmissbrauch und Cybermobbing.

Bei einem anderen medienpädagogischen Projekt, bei dem Schüler/innen ab der fünften Klasse selber Forschungsprojekte zu Medienthemen durchführten (Bündgens-Kosten 2011, Bündgens-Kosten und Kerres 2010), liess sich beobachten: Je jünger die Schüler/innen, mit denen gearbeitet wurde, desto stärker schien die Gleichsetzung von «Internet/Soziale Netzwerke» und "Gefahr». Bei älteren Jugendlichen, etwa ab der 8 . Klasse, schienen die Sorgen geringer zu sein, obwohl die Schüler/innen schon allein durch vermehrte Internetnutzung mit höherer Wahrscheinlichkeit negative Erfahrungen gemacht hatten². Trotzdem ist diese Gleichsetzung von «Sozialen Netzwerken» und «Gefahr» bis in die Mittelstufe hinein prägend. Schüler/innen in dieser Altersstufe gehen bei der Formulierung von Forschungsfragen zum Komplex «Soziale Netzwerke» wie selbstverständlich davon aus, dass diese Gleichsetzung Fakt ist, und begeistern sich sehr für Forschungsprojekte, die der Bekanntheit bestimmter Gefahrenpotenziale in ihrer Altersgruppe nachgehen, oder die die Häufigkeit von Normverstössen (z. B. Wie viele Jugendliche bestätigen Freundschaftsanfragen von Fremden/haben Fotos in ihrem Profil/geben Adresse und Telefonnummer an) erheben.

2 Livingstone et al. (2011) stellen in ihrer europäischen Stichprobe fest, dass Kinder von 9-10 Jahren zu 9\% im Vorjahr Erfahrungen gemacht hatten, die sie belastet hatten, bei den 11- bis 12-Jährigen waren dies $11 \%$, bei den 13- bis 14-Jähringen 12 Prozent, und bei den 15- bis 16-Jährigen 15\% (S. 46). 


\section{Informationsquellen zu Mediengefahren}

Woher beziehen Schüler/innen Informationen über diese Gefahren? Für die Altersgruppe 16-19 ist, nach eigenen Angaben, die Peer Group am wichtigsten (49\%), direkt gefolgt von Eltern (37\%) und Schule/Lehrer/innen (36\%). Auch die Medien (ca. 25\%) und Geschwister/Verwandte (19\%) spielen neben den eigenen Erfahrungen eine grössere Rolle (Medienpädagogischer Forschungsverbund Südwest 2010, S. 47). 77\% der Kinder und Jugendlichen in Deutschland (Altersgruppe 9-16) geben an, dass Lehrer/innen sich zum Thema "sichere Internetnutzung" in irgendeiner Form einbringen, und 95\% der Eltern von Kindern dieser Altersgruppe machen die entsprechende Aussage über sich selbst (Haddon et al. 2012, S. 26). In den folgenden Abschnitten werden wir noch einmal genauer auf die Rolle von Lehrer/innen und Schule, sowie auf den Einfluss von (Massen-)Medien bezüglich des Medienwissens von Kindern und Jugendlichen eingehen. Hierbei handelt es sich um die Informationsquellen, welche in der nachfolgenden Diskussion von Praxiskonflikten besonders relevant sein werden.

- Rolle von Schule

Die Schule ist ein Ort, an dem Medien nicht nur rezipiert, sondern auch die Nutzung bzw. der Umgang mit Medien selber thematisiert wird. Im Bildungsraum Schule werden den Schülern/-innen direkt (z. B. im Unterricht) oder indirekt (z. B. anhand der Nutzung von Medien mit und durch Mitschüler/innen) Normen vermittelt: Dabei spielen zum einen auf institutioneller Ebene u. U. eher bewahrpädagogische Ansätze eine Rolle, indem beispielsweise Handyverbote auf dem Schulhof eingeführt oder Webfilter auf den schuleigenen Computern eingerichtet werden. Zum anderen versuchen Lehrkräfte verstärkt Medien handlungsorientiert in den Unterricht zu integrieren und so den reflektierten Umgang der Schüler/innen mit Medien zu stärken (vgl. Süss et al 2010).

Auch die Projekte und Materialien, die im Verlauf des Artikels thematisiert werden, sind in aller Regel für den schulischen Einsatz konzipiert. Welchen Effekt sie haben, wird dabei sicherlich vom konkreten Einsatz im Unterricht, und damit auch von der didaktischen Planung des/der einsetzenden Lehrers/-in abhängen. Die Art etwa, wie ein konkretes Impulsvideo im Unterricht eingesetzt wird, kann ganz verschiedene medienpädagogischen Traditionen widerspiegeln: Wird es genutzt, um an Ge- und Verbote zu erinnern? Diskutieren die Schüler/innen die Aussagen, und wie sie technisch umgesetzt wurden? Gestalten die Schüler/innen selber Videos, in denen sie eigene Befürchtungen aufgreifen oder die im Impulsvideo benannten Befürchtungen karikieren? 
- Rolle von (Massen-)Medien

Auch (Massen-)Medien werden von Jugendlichen als Informationsquellen genutzt, um mehr über die Gefahren von Internet und Co zu lernen. Im europäischen Schnitt nutzen 20\% der Kinder und Jugendlichen von 9-16 Jahren Fernsehen, Radio, Zeitungen oder Zeitschriften als Quelle für Informationen zum sicheren Surfen, und 12\% beziehen entsprechende Informationen aus dem Internet, wobei diese Quellen mit zunehmenden Alter eine höhere Wichtigkeit erhalten (Livingstone et al. 2011, S. 127). Zusätzlich gilt natürlich, dass auch diejenigen Medien, die Kinder und Jugendliche nicht selber rezipieren, z. B. über Eltern oder Lehrer/innen auf die Kinder wirken können ${ }^{3}$. Dabei ist relevant, wie Medien über Computer, Internet und Co. berichten. Haddon und Stald (2009) berichten, dass in einer zweiMonats Stichprobe von Zeitungsartikeln in Deutschland zum Thema «Internet + Kinder» 40\% der insgesamt 120 Artikel über Verbrechen/Kriminalität berichteten, $26 \%$ Bildungsbezug hatten, und bei 31\% Unterhaltungsthemen dominierten. Es liegt in der Logik von Medienberichterstattung, dass einerseits Gefahren stärker betont werden als Chancen, und dass andererseits sensationelle Einzelfälle mehr Aufmerksamkeit erhalten als alltägliche Gegebenheiten. Es lässt sich also vermuten, dass (Massen-)Medien eher eine warnende, gefahrenbetonende Wirkung auf Rezipienten/-innen und deren soziales Umfeld haben.

\section{Angst und medienpädagogische Arbeit: Praxiskonflikte}

Wer mit Kindern und Jugendlichen medienpädagogisch arbeitet, agiert in einem komplexen Geflecht aus eigenen Medienerfahrungen der Schüler/innen, Medienbotschaften, sowie sozialen Einflüssen durch Peers, Eltern und Lehrer/innen. Diese verschiedenen Einflüsse sind durchaus emotional geprägt, sowohl in dem Sinne, dass Eltern oder Lehrer/innen selber eine emotionale Reaktion auf diese Themen haben, aber auch in dem Sinne, dass bestimmte Gefühle auf Seiten der Schüler/ innen evoziert oder bekräftigt werden. Die im Unterricht verwendeten Materialien sind in dieser Hinsicht ebenfalls nicht neutral. Auch hier können Gefühle, etwa Angst oder Furcht, transportiert werden.

In diesem Artikel konzentrieren wir uns auf Konflikte, Missverständnisse und Schwierigkeiten, die bei der praktischen medienpädagogischen Arbeit auftreten können, und die im weitesten Sinne mit Angst oder Gefahrenbewusstsein zusammenhängen. Vier Aspekte werden dabei besonders thematisiert: Rolle von Angst und Scham, Gefahrenbewusstsein und Handlungsempfehlungen, Verzerrte Gefahrenwahrnehmung sowie Fokus auf Extremfälle. Dies erhebt natürlich nicht den

3 Im europäischen Schnitt beziehen 32\% der Eltern ihre Informationen zur Internetsicherheit, und damit auch zu den Gefahren Sozialer Netzwerke, aus TV, Radio, Zeitungen und Zeitschriften, 21\% aus dem Netz selbst (Livingstone et al. 2011, S. 128). Die Wahrscheinlichkeit, dass 68\% der Eltern, die angaben, aus TV und Co keine Informationen zu diesem Thema zu beziehen, trotzdem darauf bezogene Inhalte (etwa in Nachrichten oder Boulevardsendungen, aus Plotelementen in Filmen, etc.) aufgenommen haben, ist jedoch gross. 
Anspruch auf Vollständigkeit - besonders die Herausforderungen einer stärker gestaltenden Medienarbeit fehlen an dieser Stelle - es werden aber einige der von den Autorinnen häufig beobachteten Probleme adressiert.

\section{Rolle von Angst und Scham}

Ein Bewusstsein für die Gefahrenpotenziale von Sozialen Netzwerken sowie ein belastbares Wissen über deren Vermeidung oder Minimierung ist sicherlich wünschenswert. Aber was, wenn Gefahrenbewusstsein zur Angst wird? Oder noch pointierter: Sollten Medienpädagogen/-innen versuchen, Angst zu schüren?

Der Ansatz ist aus der Verkehrserziehung bekannt: Um Gefahren von Alkohol am Steuer zu verdeutlichen, werden etwa Unfallszenen verwendet. Der Appell an Angst ('fear appeal〉(Barth und Bengel 2000)), Ekel oder sonstige basale Gefühle dient dazu, bestimmtes Verhalten zu stärken oder zu schwächen. Ähnliche AngstAppelle sind auch aus der gesundheitlichen Aufklärung (z. B. AIDS, Rauchen, binge drinking, etc.) bekannt.

Auch für die medienpädagogische Arbeit gibt es viele Ressourcen, die Gefühle - meist Angst oder Scham - ansprechen sollen ${ }^{4}$. Beispiele für ersteres sind z. B. der WatchYourWeb Spot «Masken»5. Der Kurzfilm zeigt ein Mädchen, das im Park spazieren geht. Dort trifft sie auf ihre Online-Bekanntschaften in Form von maskierten Gestalten, zu denen auch Stellvertreterfiguren für Cybermobbing und Cyber-Grooming gehören, und denen sie nicht entkommen kann. Das Mädchen bekommt Angst und versucht, vor den maskierten Gestalten zu fliehen. Erst der «Internet-Superheld» Watchman kann ihr helfen. Wie eine solche Hilfe bei Cybermobing oder Cyber-Grooming konkret aussehen könnte, wird nicht gezeigt, der im Spot gezeigte Lösungsansatz bewegt sich auf einer rein symbolischen Ebene: In einer animierten Szene kämpft Watchman gegen seinen Rivalen Data Devil, den er schliesslich besiegt. Begleitet wird diese animierte Szene durch den Spruch «Watch your web». Der Handlungsstrang, in dem das Mädchen durch maskierte Figuren bedroht wurde, wird letztlich nicht aufgelöst. Insgesamt hat der Spot eine sehr düstere, angsteinflössende Atmosphäre, konkrete Handlungsempfehlungen werden im Spot nicht gegeben.

Andere Beispiele für Videos, die bewusst Angst oder Scham einsetzten, sind etwa «Date» ${ }^{6}$, auch von WatchYourWeb, oder das norwegische Video «Lehrerkonferenz»", von klicksafe.de empfohlen. Beide setzen äusserlich auf Humor, aber in der Filmhandlung selbst wird das klassische erzieherische Mittel der Blossstellung bzw.

4 Entscheidend ist natürlich nicht die kommunikative Absicht oder die Einschätzung der Wirkung durch ein Fachpublikum, sondern die tatsächliche Wirkung auf Kinder und Jugendliche, die sich davon durchaus unterscheiden kann.

5 http://www.watchyourweb.de/p168735295_450.html

6 http://www.watchyourweb.de/p3548375992_450.html\#film_anschauen

7 http://www.klicksafe.de/ueber-klicksafe/downloads/weitere-spots/norwegen-lehrerkonferenzdeutsch 
Beschämung eingesetzt: Bei «Date», indem ein Junge auf einem Spaziergang mit einem Mädchen durch öffentlich sichtbare Fotos (auf Plakatwand, Flyer, etc.), die seine früheren Beziehungen dokumentieren, in Verlegenheit gebracht wird, bei «Lehrerkonferenz», indem ein Schüler im Beisein seiner Eltern durch seine Lehrerin mit seinen Onlineaktivitäten (Fotos, beleidigende Blogbeiträge) konfrontiert wird. Auch medienpädagogischen Materialien und Aktivitäten, die bewusst darauf setzen, Schüler/innen mit Rückgriff auf ihr bisheriges Medienhandeln blosszustellen, etwa indem kompromittierende Bilder der jeweiligen Schüler/innen in Sozialen Netzwerken recherchiert und innerhalb einer Klassencommunity präsentiert werden oder indem gezeigt wird, wie viele personenbezogene Daten über sie verfügbar sind, nutzen Angst und Scham.

Aus der Forschung zur gesundheitlichen Aufklärung ist bekannt, dass Angst durchaus zu erwünschten Verhaltensänderungen führen kann, auch wenn noch nicht endgültig geklärt ist, ob stärkere Angst einen stärkeren Effekt hat, oder zumindest unter bestimmten Umständen zu einer Ablehnung der vermittelten Botschaft führt. Gleichzeitig ist auch bekannt, dass angsterzeugende Nachrichten immer mit Handlungsmöglichkeiten gekoppelt werden sollten, um tatsächlich zu Verhaltensänderungen führen zu können (Barth und Bengel, 2000).

Es ist natürlich zu fragen, ob diese Erkenntnisse eins zu eins auf medienpädagogische Kontexte übertragbar sind - und ob wir sie auf diese Kontexte übertragen wollen. Welche Rolle Angst - über ein angemessenes Gefahrenbewusstsein hinaus - spielen soll, hängt sicherlich von unseren Annahmen über die Rolle von Angst in Erziehung und Bildung allgemein sowie von ethischen Grundüberlegungen ab. Gleiches gilt für Scham und Blossstellung.

Sicher ist jedoch, dass Emotionen wie Angst oder Scham keinesfalls geweckt werden sollten, ohne dass anschliessend konkrete (und realistische!) Handlungsmöglichkeiten diskutiert werden. Sonst ist die Folge kein bedachterer Umgang mit dem Netz, sondern eher Panik und das Ignorieren von möglichen Problemen (Livingstone et al. sprechen in diesem Zusammenhang von einer fatalistischen (fatalistic) (im Gegensatz zu einer proaktiven (pro-active) oder einer selbstbeschuldigenden (self-accustory)) Reaktion (Livingstone et al 2011, S. 59)). Dann sind Angst und Scham in jedem Fall schlechte Ratgeber.

Im nächsten Abschnitt werden wir auf Handlungsempfehlungen noch einmal gesondert eingehen.

\section{Gefahrenbewusstsein und Handlungsempfehlungen}

Wie schon oben diskutiert, haben praktisch alle Kinder und Jugendlichen ein Bewusstsein für mögliche Gefahren in Sozialen Netzwerken, in der Regel können sie auch konkrete Handlungsempfehlungen benennen. Dies betrifft nicht nur Nutzer/ innen Sozialer Netzwerke. Selbst Kinder, die nach eigenen Angaben nie oder fast 
nie online sind, haben von Fake Accounts, Cybermobbing und Facebook Parties gehört und können Handlungsempfehlungen, die ihnen durch Familie, Schule oder Medien vermittelt wurden, wiedergeben. Oft sind diese Handlungsempfehlungen - unabhängig vom Alter - in absolute Formulierungen gekleidet: «Niemals persönliche Daten im Internet preisgeben», «keine Fotos auf Facebook einstellen», «immer unter Pseudonym agieren». Während jüngere Schüler/innen solchen Empfehlungen - ggf. unter enger Begleitung durch die Eltern - eher folgen, haben viele Jugendliche einen pragmatischeren Umgang mit dem Netz entwickelt. Einerseits sind ihnen die ‘absoluten〉 Regeln vermittelt worden, andererseits haben sie die praktische Erfahrung gemacht, dass diese Regeln vereinfacht sind und der Komplexität der sozialen Interaktionen im Netz nicht entsprechen.

Die Schüler/innen haben dann zwar die soffiziellens Regeln, d. h. die Normen und Vorgaben, gespeichert und geben sie auf Nachfrage wieder, ihr Verhalten richten sie aber nach komplexeren, oft unausgesprochenen Regeln aus. Hier ist ein Widerspruch zwischen Handeln und öffentlich gegenüber Lehrern/-innen oder Eltern vertretener Meinung zu erkennen (vgl. ¿Cognitive Dissonance), Festinger et al 1956). Fragt man nun in einem Unterrichtsgespräch nur danach, wie man sich im Netz verhalten soll, ist es möglich, dass diese Differenz zwischen wiedergegebener «Lehrmeinung» und dem eigenen Verhalten nicht zur Sprache kommt. So entgehen viele Möglichkeiten, die vielleicht sogar sinnvollen impliziten Regeln zu diskutieren, zu reflektieren und gemeinsam mit den Schülern/-innen auf Basis gemachter Erfahrungen Schwachstellen zu identifizieren und zu korrigieren. Zumindest sollten, wenn es nicht ratsam erscheint, explizit nach Regelverletzungen zu fragen, praktische Probleme beim Umsetzen dieser Standards angesprochen werden. Der Fokus sollte dabei auf tatsächlichem Verhalten liegen, nicht auf dem Abspeichern und der Wiedergabe der mit diesem Verhalten nicht notwendigerweise verknüpften Verhaltensnormen.

Ein gewisses Augenmerk verdient auch das Phänomen, dass zwar Lehrer/innen ihre Schüler/innen vermehrt auffordern, AGBs zu lesen, und etwa den Zugang zu bestimmten Medien aufgrund von AGBs (Stichwort: Altersbeschränkung) u.ä. zu regulieren versuchen, gleichzeitig aber Schüler/innen u. U. auffordern, sich entgegen AGBs mit falschem Namen anzumelden, oder z. B. selber entgegen AGBs SchülerVZ-Profile unterhalten. Hier entsteht ebenfalls ein Widerspruch zwischen verschiedenen Handlungsempfehlungen, bzw. zwischen Handlungsempfehlungen und beobachtbarem Verhalten.

\section{Verzerrte Gefahrenwahrnehmung}

Hin und wieder erhalten einzelne mit dem Internet oder Sozialen Netzwerken verbundene Gefahren besondere Aufmerksamkeit. Diese Aufmerksamkeit, die etwa durch die Häufigkeit von Medienberichterstattung oder die Dringlichkeit von 
Forderungen nach gesetzlicher Regulierung sichtbar wird, spiegelt dabei nicht notwendigerweise ein tatsächliches hohes Risiko wieder. Man denke in diesem Kontext etwa an die Berichterstattung über den 〈Trend Facebookparty〉, zu einem Zeitpunkt, an dem es in Deutschland erst einen einzigen Vorfall dieser Art gegeben hatte.

Medienaufmerksamkeit kann, wie oben ausgeführt, auch das Gefahrenbewusstsein, die Gefahreneinschätzung, und ggf. auch Angst auf Schüler/innenseite beeinflussen.

In ihrer medienpädagogischen Arbeit sind die Autorinnen oft mit starken Ängsten auf Schüler/innenseite konfrontiert worden. Gerade bei Schülern/-innen der Unterstufe, die oft noch keine oder sehr geringe Erfahrungen mit Sozialen Netzwerken haben, spielt die Sorge vor Cybermobbing durch vollkommen unbekannte Personen, oder die Gefahr durch Stalker/innen und Hacker/innen ${ }^{8}$ beim Anlegen eines Facebook Profils, eine grosse Rolle. Andere, alltäglichere Gefahren dagegen, wie Mobbing durch bekannte Personen, oder der Missbrauch anvertrauter Passwörter, geniessen bei dieser Altersgruppe weniger Aufmerksamkeit.

Verzerrte Gefahrenwahrnehmung, primär von jüngeren Schülern/-innen, kann Akteure/-innen bei der medienpädagogischen Arbeit in die schwierige Situation bringen, dass man einerseits für die Gefahren der Netznutzung sensibilisieren und die Sorgen der Schüler/innen ernst nehmen möchte, andererseits aber vielleicht einen breiteren Fokus der Aufmerksamkeit für sinnvoller hält. Ein konkretes Interesse an z. B. Facebookparties kann sicherlich genutzt werden, um ganz allgemein über Privatssphäreneinstellungen oder Netzkultur zu sprechen, die Angst vor «Hackern/-innen» kann zu einem Gespräch über die Wahl sicherer Passwörter und die Rolle von Social Engineering Anlass geben. Eine Beschränkung auf die aktuelle «Mode-Sorge» selber scheint aber nur bedingt sinnvoll.

\section{Fokus auf Extremfälle}

Oft lässt sich in den Diskussionen mit Schülern/-innen ein Fokus auf Extremfälle beobachten. Dieser Fokus auf Extremfälle kann zu der oben geschilderten Verzerrung der Gefahrenwahrnehmung beitragen, ist aber auch unabhängig davon problematisch und sollte als getrenntes Phänomen Beachtung finden.

Was genau mit einem Fokus auf Extremfällen gemeint ist, lässt sich am Besten durch ein Beispiel erläutern: In einem Gespräch über Soziale Netzwerke erklärte eine Schülerin der Unterstufe, dass soziale Netzwerke ein wichtiges Thema seien, da sie Cybermobbing hervorriefen, was wiederum zu Selbstmord führe. Mobbing,

8 Dass Schüler/innen besonders vor Stalkern/-innen und Hackern/-innen Angst hätten, war u.a. das überraschende Ergebnis einer im kleinen Rahmen durchgeführte Befragung von UnterstufenSchülern/-innen durch andere Unterstufen-Schüler/innen. Aber auch in Gesprächen wurde oft der Sorge Ausdruck verliehen, fremde Personen könnten sich z. B. in Facebook-Accounts seinhacken». Der Missbrauch fremder Facebook-Accounts ist leider in vielen Fällen Realität, dabei handelt es sich jedoch oft um den Missbrauch anvertrauter Login-Daten, nicht um ein klassisches «Einhacken`. 
egal ob online oder offline, ist soziale Realität und hat oft dramatische Folgen. Aber weder mediale Berichterstattung noch medienpädagogische Initiativen tun den Opfern einen Gefallen, wenn sie sich nur auf die Extremfälle konzentrieren. Auch in den von aussen weniger dramatisch wirkenden Fällen ist Cybermobbing problematisch. Je extremer das Problem in den Medien dargestellt wird, desto weniger beziehen Kinder und Jugendliche das Berichtete auf sich. Aussagen der Art «Nur, wenn ich einen Facebook Account knacke, mich als jemand anderes ausgebe und so alle seine Freundschaften ruiniere, nur dann betreibe ich «Cybermobbing»» oder "Nur wenn ich eine Fake Account betreibe, um jemanden fertig zu machen, cybermobbes ich», stehen für dieses Verständnis von Cybermobbing als absolute Extremform des digitalen Mobbings?.

Wagner et al. (2012) haben untersucht, wie Jugendliche Konflikte in Sozialen Netzwerken wahrnehmen. Sie zeigen auf, dass Kinder und Jugendliche ein Verständnis von Cybermobbing haben, das zum Teil stark von dem der (medienpädagogisch tätigen) Erwachsenen abweicht. Oft wird unter Cybermobbing das wiederholte oder systematische Ärgern, Blossstellen, Beleidigen einer Person durch andere verstanden, wobei in den Diskussionen über Cybermobbing der Faktor «wiederholt oder systematisch〉 teilweise vernachlässigt wird, und der Fokus dadurch auf konkrete, als nicht akzeptabel verstandene, Handlungen (etwa beleidigende Fotomontagen ins Netz stellen, Hassgruppen gründen, Beleidigungen auf Facebook Wall schreiben, koordiniertes Un-Friending) gelegt wird ${ }^{10}$. Für Schüler/innen dagegen spielt die Intensität der Handlungen in Relation zum eigenen Wertesystem und bezogen auf den konkreten Einzelfall eine zentrale Rolle. Dabei wird abhängig von der Situation und den beteiligten Gruppen und Personen entschieden, ob von «Mobbing) die Rede sein kann: «(Cyber-)Mobbing wird von den Jugendlichen als Sammelbegriff für ein aggressives Konflikthandeln gebraucht, das nach dem eigenen moralischen Verständnis zu weit geht.» (ebd. S. 28). Die Gleichsetzung von Cybermobbing mit konkreten, aber entkontextualisierten Handlungen, widerspricht diesem Verständnis vieler Jugendlicher.

Dieses unterschiedliche Verständnis von Cybermobbing zeigt sich auch bei der praktischen medienpädagogischen Arbeit. Bei einem Projekt, bei dem Schüler/innen selber Forschungsprojekte zu Medienthemen durchführten, haben viele Schüler/innengruppen Studien zur Häufigkeit von Cybermobbing durchgeführt. Ergebnisse, die auf eine äusserst geringe Häufigkeit von Cybermobbing an einer Schule hinweisen, müssen dabei nicht Folge von sozialer Erwünschtheit sein - sie können

9 Man beachte, wie hier die Sorge vor Cybermobbing oft mit der Angst vor Hackern/-innen und Fakes verbunden ist.

10 Vgl. hier Wolak, Mitchell und Finkelhor's (2007) Unterscheidung zwischen «harrassment» und «bullying». 
auch durch ein rein sprachliches Missverständnis erklärt werden ${ }^{11}$. Von einer solchen Statistik, die auf eine Klärung des Begriffes «Cybermobbing` weitestgehend verzichtet, ist jedenfalls nicht direkt auf die Verbreitung bestimmter Handlungen bzw. auf die jeweilige Bewertung dieser Handlungen durch die Schüler/innen zu schliessen.

Bei medienpädagogischer Arbeit kann es also sinnvoll sein, die gemeinsam verwendeten Begriffe zu klären. Was verstehen die Schüler/innen unter «typischem Cybermobbing»? Was meinen die beteiligten Lehrer/innen oder Medienpädagogen/innen mit diesem Begriff? So können einerseits Missverständnisse vermieden werden, andererseits kann der Fokus auf durchaus belastende, wenn auch im Vergleich zu manchen Cybermobbing-Fällen harmlos anmutende Formen von ¿Stress〉 gelenkt werden. Denn die Auseinandersetzungen von Schülern/-innen auf Facebook können auch schon da belastend sein, wo das Schüler/innenverständnis von ¿Cybermobbing» noch nicht erreicht wurde.

Darüber hinaus ist zu überlegen, in wie fern dramatische, spannende Einzelfälle tatsächlich optimale Aufhänger für die mediendidaktische Arbeit an Schulen sind. Livingstone et al. (2011) etwa fordern: «Parents need to be alerted to the risks involved while avoiding an alarmist or sensationalist approach.» (S. 145, Fettdruck im Original). Diese Forderung lässt sich so auch auf die Arbeit mit Kindern und Jugendlichen übertragen: Risiken sollten bewusst gemacht werden, aber Alarmismus und Sensationalisierung sind dazu nicht der optimale Weg. Ein vollständiger Verzicht auf die Behandlung von Extremfällen wird kaum möglich sein, da die Schüler/innen solche Beispiele oft selber anführen. Es ist jedoch sicherlich möglich, durch eine Reihe von Beispielen, von denen einige auch banal oder alltäglich zu sein scheinen, eine gewisse Balance zu erreichen.

\section{Schlussfolgerung}

In diesem Artikel haben wir einige Praxiskonflikte, die in der medienpädagogischen Arbeit im Zusammenhang mit Gefahreneinschätzung und damit verbundenen Medienängsten entstehen können, vorgestellt.

Angst - auf Seiten der Schüler/innen, aber ebenso auf Lehrer/innen- oder Elternseite - kann ein mächtiger Katalysator sein. Sie schafft eine hohe Sensibilität gegenüber einem Problem, und oft auch erst den Handlungsdruck, der notwendig ist, um trotz zentraler Abschlussprüfungen und ständig wachsender Ansprüche an die Schule Raum zu schaffen für medienpädagogische Interventionen, vom spon-

11 Ein ähnliche überraschendes Ergebnis einer von Schülern/-innen im Rahmen des gleichen Projekts durchgeführten Studie: Viele Schüler/innen einer Schule fanden Cybermobbing unproblematisch. Hier ist zu vermuten, dass Schüler/innen ein Erwachsenenverständnis (etwa «Cybermobbing ist jegliche Art von Beleidigungen online») übernommen hatten. Aller Wahrscheinlichkeit nach begrüssten sie nicht das systematische Fertigmachen einer Person weit über die persönliche Moralgrenze hinaus, sondern waren mit Spass-Streitigkeiten (vgl. Wagner et al. 2012, 26) und auch mit etwas barscheren Mitteln als Werkzeug bei ‘Stress〉 durchaus einverstanden. 
tanen Klassengespräch bis hin zum grösseren medienpädagogischen Projekt. So entstehen spannende Initiativen und Projekte auf Klassen- oder Schulebene, bevor es notwendigerweise zu grösseren Vorfällen an der Schule gekommen sein muss. Ob man Angst auf Schüler/innenseite bewusst schüren sollte, etwa durch den Einsatz potenziell angsterzeugender Materialien und Aktivitäten, darüber mag man geteilter Meinung sein. Klar ist aber, dass das Gewicht nicht auf der Warnung vor Gefahren liegen sollte, die den Schülern/-innen oft schon bewusst sind, sondern eher darauf, angemessene Reaktionen auf diese Gefahren zu entwickeln.

Gerade ein Fokus auf sensationelle, tragische, schockierende Vorfälle schafft zwar Aufmerksamkeit und Betroffenheit, aber die Übertragung dessen, was man am Spezialfall gelernt hat, auf den alltäglicheren Kontext, fällt Schülern/-innen nicht immer leicht. Wenn man dennoch sensationelle Beispiele einbringen möchte, oder diese den Schülern/-innen z. B. aus der Medienberichterstattung bereits bekannt sind, sollte man diese immer mit alltäglichen Beispielen ergänzen. Bei der Diskussion über z. B. Indikatoren und Hilfemöglichkeiten, über Parallelen und feine Unterschiede zwischen Szenarien, gilt es besonders, verschiedene Beispiele explizit zum Thema machen.

Auch, um glaubwürdig zu bleiben, ist ein solch differenzierterer Umgang mit Mediengefahren sinnvoll. Je mehr Erfahrung die Schüler/innen mit einem OnlineAngebot haben, desto essentieller ist eine differenzierte Kritik, die nicht über die praktischen Erfahrungen der Schüler/innen hinweggeht.

Klar ist: Angst, Sorgen, Befürchtungen auf Seiten von Schüler/innen, Eltern, Lehrer/innen, sicherlich auch auf Seiten von Medienpädagogen/-innen selber, sind vorhanden. Sie zu ignorieren, ist keinesfalls sinnvoll. Bei medienpädagogischer Arbeit geht es nicht nur um Faktenwissen und Handlungsanleitung - medienpädagogische Arbeit hat auch eine affektive Dimension, der man sich in der praktischen Arbeit stets bewusst sein sollte.

\section{Literatur}

Barth, Jürgen und Jürgen Bengel. 2000. «Prevention through fear? The state of fear appeal research.» Köln: BZgA.

Bündgens-Kosten, Judith und Tobias Hölterhof. 2013. «Angst vor dem Netz. Medienangst und pädagogisches Handeln.» Kinder- und Jugendschutz 2: 58-61.

Bündgens-Kosten, Judith und Michael Kerres. 2010. "Öffnung von Hochschule: Auch für Kinder und Jugendliche?» Hochschulwesen: Forum für Hochschulforschung, -praxis und -politik 4+5: 155-159.

Bündgens-Kosten, Judith. 2011. «Vermittlung von Medienkompetenz im Pädagogikunterricht: Das Beispiel «Schülerkolleg Pädagogik»»» PädagogikUNTERRICHT 4: 57-62. 
Cohen, Stanley. 1987. Folk Devils and Moral Panics: The Creation of the Mods and Rockers. Oxford: Basil Blackwood.

Drotner, Kirsten. 1992. «Modernity and Media Panics.» In Media Cultures: Reappraising Transnational Media, ed. Michael Skovmand and Kim Schrøder, 42-62. London: Routledge.

Festinger, Leon, Henry W. Riecken and Stanley Schachter. 1956. When Prophecy Fails: A Social and Psychological Study of a Modern Group that Predicted the Destruction of the World. New York: Harper-Torchbooks.

Haddon, Leslie and Gitte Stald. 2009. "Comparative Analysis of European Press Coverage of Children and the Internet.» Journal of children and media 3 (4): 379-393.

Keuneke, Susanne. 2011. «Medienangst als Massstab: Der wechselhafte Umgang mit dem Populären am Beispiel der Literatur.» In Kommunikation im Populären: Interdisziplinäre Perspektiven auf ein ganzheitliches Phänomen. hrsg. v. Roger Lüdeke, 273-296. Bielefeld: transcript Verlag.

Haddon, Leslie, Sonia Livingstone and the EU Kids Online network EU kids. online: national perspectives 2012.

Livingstone, Sonia, Leslie Haddon, Anke Görzig and Kjartan Ólafsson. 2011. Risks and Safety on the Internet: The perspective of European children. Full Findings. London: EU Kids Online.

Marwick, Alice E. 2008. «To catch a predator? The MySpace moral panic.» First Monday 13 (6): http://firstmonday.org/htbin/cgiwrap/bin/ojs/index.php/fm/ article/view/2152/1966.

Medienpädagogischer Forschungsverbund Südwest. 2011. JIM-Studie 2011: Jugend, Information, (Multi-) Media. Basisuntersuchung zum Medienumgang 12bis 19-Jähriger. Stuttgart: mpfs. www.mpfs.de/fileadmin/JIM-pdf11/JIM2011.pdf.

Medienpädagogischer Forschungsverbund Südwest. 2010. JIM-Studie 2010: Jugend, Information, (Multi-) Media. Basisuntersuchung zum Medienumgang 12bis 19-Jähriger. Stuttgart: mpfs. www.mpfs. de/fileadmin/JIM-pdf10/JIM2010.pdf.

Süss, Daniel, Claudia Lampert und Christine W. Wijnen. 2010. Medienpädagogik: Ein Studienbuch zur Einführung. Wiesbaden: VS Verlag für Sozialwissenschaften.

Wagner, Ulrike, Niels Brüggen, Peter Gerlicher und Mareike Schemmerling. 2012. "Wo der Spass aufhört... Jugendliche und ihre Perspektive auf Konflikte in Sozialen Netwerkdiensten. Teilstudie im Projekt «Das Internet als Rezeptions- und Präsentationsplattform für Jugendliche.» Hrsg. v. der Bayerischen Landeszentrale für neue Medien (BLM). München: JFF - Institut für Medienpädagogik in Forschung und Praxis: http://www.jff.de/dateien/JFF-Bericht_Online-Konflikte.pdf.

Wolak, Janis, Kimberly Mitchell und David Finkelhor. 2007. «Does online harassment constitute bullying? An exploration of online harassment by known peers and online-only contacts». Journal of adolescent health 41 (6): 51-58. 